\title{
Extensions of Boolean Rings and Nearrings
}

\author{
Hamsa Nayak \\ Syam P. Kuncham \\ Babushri S. Kedukodi* \\ Department of Mathematics \\ Manipal Institute of Technology \\ Manipal Academy of Higher Education \\ Manipal Karnataka, 576104 \\ India
}

Received 15.06.2018, received in revised form 06.09.2018, accepted 01.12.2018

\begin{abstract}
In this paper, we introduce the notions of left (right) Boolean rings and nearrings. We give examples to show that left (right) Boolean rings are not commutative in general. We obtain interrelations among these algebraic structures and get conditions under which the structures are commutative. Finally, we study the concept of derivations on left (right) Boolean rings and nearrings and obtain commutativity results.
\end{abstract}

Keywords: ring, nearring, Boolean ring, Boolean nearring.

DOI: $10.17516 / 1997-1397-2019-12-1-58-67$.

\section{Introduction}

A nearring $(N,+, \cdot)$ is an algebraic system with binary operations addition and mutiplication satisfying the axioms of a ring, except commutativity of addition and one of the distributive laws. Right nearrings satisfy the right distributive law and left nearrings satisfy the left distributive law. A natural example of right nearring is the set of all mappings from a group $(G,+)$ to itself under addition and composition of mappings. In this sequel, $N$ denotes a right nearring.

A Boolean ring $R$ is a ring for which $x^{2}=x$ for all $x \in R$. An example of Boolean ring is the ring of integers modulo 2. If all the elements in a nearring are idempotent, then such a nearring is called a Boolean nearring. Clay and Lawyer [7] showed that a distributively generated Boolean nearring is multiplicatively commutative by using subdirect sum representations of nearrings. Reddy [27] studied recent developments in Boolean nearrings. Bhavanari and Kuncham [2] introduced the concepts of linearly independent elements and u-linearly independent elements in N-group. Bhavanari, Godloza, Babu and Kuncham [6] introduced zerosquare nearring of type-1/type-2, their ideals, zero square dimension of a nearring and obtained several results. Bhavanari, Kuncham and Kedukodi [5] studied graph theoretic aspects of nearrings. Kedukodi, Kuncham and Bhavanari [12,13] studied equiprime, 3-prime and c-prime fuzzy ideals of nearrings. Jagadeesha, Kedukodi, Kuncham [9, 16, 19] studied homomorphic images of interval valued Lfuzzy ideals and proved isomorphism theorems. Molkhasi [22] defined the notion of strongly algebraically closed lattices.

For recent developments in nearrings and Boolean nearrings, we refer Kuncham, Kedukodi, Panackal and Bhavanari [20], Nayak, Kuncham and Kedukodi [23], Koppula, Kedukodi and Kuncham [17] and Kedukodi, Kuncham and Bhavanari [14,15]. We refer Bhavanari and Kuncham [3],

\footnotetext{
*babushrisrinivas.k@manipal.edu

(c) Siberian Federal University. All rights reserved
} 
Kuncham [18], Pilz [24], Bhavanari [4], Lafuerza-Guillén and Panackal [21], Panackal, LafuerzaGuillén and Ravindran [25] and Jagadeesha, Kuncham and Kedukodi [8] for basic definitions. The paper is divided into two sections. In Section 1, we introduce left, right Boolean rings and nearrings. We give conditions under which the structures are weak commutative with respect to an ideal. In Section 2, we study derivations on left and right Boolean ring and nearring.

\section{Left and right Boolean rings and nearrings}

Definition 1.1. Let $N$ be a nearring. $N$ is called a left (resp. right) Boolean nearring if there exists $n \in N$ such that $x^{2}=n x$ (resp. $x^{2}=x n$ ) for all $x \in N$. If $N$ is a ring satisfying $x^{2}=n x$ (resp. $\left.x^{2}=x n\right)$ for all $x \in N$ then $N$ is called a left (resp. right) Boolean ring.

We begin with an example of left (resp. right) Boolean ring which is not a Boolean ring.

Example 1.2. Let $R=\{0, a, b, c\}$ with operations + and $\cdot$ as follows:

\begin{tabular}{|c|c|c|c|c|}
\hline+ & 0 & $a$ & $b$ & $c$ \\
\hline 0 & 0 & $a$ & $b$ & $c$ \\
\hline$a$ & $a$ & 0 & $c$ & $b$ \\
\hline$b$ & $b$ & $c$ & 0 & $a$ \\
\hline$c$ & $c$ & $b$ & $a$ & 0 \\
\hline
\end{tabular}

\begin{tabular}{|c|c|c|c|c|}
\hline$\cdot$ & 0 & $a$ & $b$ & $c$ \\
\hline 0 & 0 & 0 & 0 & 0 \\
\hline$a$ & 0 & 0 & 0 & 0 \\
\hline$b$ & 0 & 0 & $b$ & $b$ \\
\hline$c$ & 0 & 0 & $b$ & $b$ \\
\hline
\end{tabular}

Then $R$ is a left as well as right Boolean ring for $n \in\{b, c\}$. We have $a^{2}=0 \neq a$. Hence $R$ is not a Boolean ring.

Example 1.3. Let $R$ be a left(resp. right) Boolean ring. In $R^{3}$, define addition componentwise and multiplication by $\left(x_{1}, y_{1}, z_{1}\right)\left(x_{2}, y_{2}, z_{2}\right)=\left(0,0, x_{1} y_{2}-x_{2} y_{1}\right)$. Then $R^{3}$ is a left(resp. right) Boolean ring.

Verification: Let $\left(x_{1}, y_{1}, z_{1}\right),\left(x_{2}, y_{2}, z_{2}\right) \in R^{3} .\left(x_{1}, y_{1}, z_{1}\right)\left(x_{2}, y_{2}, z_{2}\right)=\left(0,0, x_{1} y_{2}-x_{2} y_{1}\right)$ and $\left(x_{2}, y_{2}, z_{2}\right)\left(x_{1}, y_{1}, z_{1}\right)=\left(0,0, x_{2} y_{1}-x_{1} y_{2}\right)$. Clearly, $\left(x_{1}, y_{1}, z_{1}\right)\left(x_{2}, y_{2}, z_{2}\right)+\left(x_{2}, y_{2}, z_{2}\right)\left(x_{1}, y_{1}, z_{1}\right)=$ $=(0,0,0)$.

We show that distributive properties are satisfied:

Let $\left(x_{1}, y_{1}, z_{1}\right),\left(x_{2}, y_{2}, z_{2}\right),\left(x_{3}, y_{3}, z_{3}\right) \in R^{3}$.

$$
\begin{array}{r}
\left(x_{1}, y_{1}, z_{1}\right)\left(\left(x_{2}, y_{2}, z_{2}\right)+\left(x_{3}, y_{3}, z_{3}\right)\right)=\left(x_{1}, y_{1}, z_{1}\right)\left(x_{2}+x_{3}, y_{2}+y_{3}, z_{2}+z_{3}\right)= \\
=\left(0,0, x_{1}\left(y_{2}+y_{3}\right)-\left(x_{2}+x_{3}\right) y_{1}\right)=\left(0,0, x_{1} y_{2}+x_{1} y_{3}-x_{2} y_{1}-x_{3} y_{1}\right) .
\end{array}
$$

Now,

$$
\begin{array}{r}
\left(x_{1}, y_{1}, z_{1}\right)\left(x_{2}, y_{2}, z_{2}\right)+\left(x_{1}, y_{1}, z_{1}\right)\left(x_{3}, y_{3}, z_{3}\right)=\left(0,0, x_{1} y_{2}-x_{2} y_{1}\right)+\left(0,0, x_{1} y_{3}-x_{3} y_{1}\right)= \\
=\left(0,0, x_{1} y_{2}-x_{2} y_{1}+x_{1} y_{3}-x_{3} y_{1}\right) .
\end{array}
$$

Hence $\left(x_{1}, y_{1}, z_{1}\right)\left(\left(x_{2}, y_{2}, z_{2}\right)+\left(x_{3}, y_{3}, z_{3}\right)\right)=\left(x_{1}, y_{1}, z_{1}\right)\left(x_{2}, y_{2}, z_{2}\right)+\left(x_{1}, y_{1}, z_{1}\right)\left(x_{3}, y_{3}, z_{3}\right)$. Similarly we can verify that

$$
\left(\left(x_{1}, y_{1}, z_{1}\right)+\left(x_{2}, y_{2}, z_{2}\right)\right)\left(x_{3}, y_{3}, z_{3}\right)=\left(x_{1}, y_{1}, z_{1}\right)\left(x_{3}, y_{3}, z_{3}\right)+\left(x_{2}, y_{2}, z_{2}\right)\left(x_{3}, y_{3}, z_{3}\right) .
$$

Now, we show that the associative law is satisfied.

$$
\left(x_{1}, y_{1}, z_{1}\right)\left(\left(x_{2}, y_{2}, z_{2}\right)\left(x_{3}, y_{3}, z_{3}\right)\right)=\left(x_{1}, y_{1}, z_{1}\right)\left(0,0, x_{2} y_{3}-x_{3} y_{2}\right)=(0,0,0) .
$$

Hence $\left(\left(x_{1}, y_{1}, z_{1}\right)\left(x_{2}, y_{2}, z_{2}\right)\right)\left(x_{3}, y_{3}, z_{3}\right)=\left(0,0, x_{1} y_{2}-x_{2} y_{1}\right)\left(x_{3}, y_{3}, z_{3}\right)=(0,0,0)$. Now, we show that $R^{3}$ is a left(resp. right) Boolean ring. We have $(x, y, z)(x, y, z)=(0,0, x y-x y)=$ $=(0,0,0)=(x, y, z)(0,0, n)=(0,0, n)(x, y, z)$. 
Example 1.4. Let $M=\left\{\left[\begin{array}{ll}x & 0 \\ 0 & x\end{array}\right] \mid x \in N\right\}$, where $N=\{0, a, b, c\}$ and + and $\cdot$ are defined on $N$ as follows:

\begin{tabular}{|c|c|c|c|c|}
\hline+ & 0 & $a$ & $b$ & $c$ \\
\hline 0 & 0 & $a$ & $b$ & $c$ \\
\hline$a$ & $a$ & 0 & $c$ & $b$ \\
\hline$b$ & $b$ & $c$ & 0 & $a$ \\
\hline$c$ & $c$ & $b$ & $a$ & 0 \\
\hline
\end{tabular}

\begin{tabular}{|c|c|c|c|c|}
\hline$\cdot$ & 0 & $a$ & $b$ & $c$ \\
\hline 0 & 0 & 0 & 0 & 0 \\
\hline$a$ & 0 & 0 & 0 & 0 \\
\hline$b$ & 0 & 0 & 0 & $a$ \\
\hline$c$ & 0 & 0 & 0 & $a$ \\
\hline
\end{tabular}

Then $M$ is a left Boolean nearring with $n=\left[\begin{array}{ll}c & 0 \\ 0 & c\end{array}\right]$ because

$$
\left[\begin{array}{ll}
x & 0 \\
0 & x
\end{array}\right]\left[\begin{array}{ll}
x & 0 \\
0 & x
\end{array}\right]=\left[\begin{array}{cc}
x^{2} & 0 \\
0 & x^{2}
\end{array}\right]=\left[\begin{array}{cc}
c x & 0 \\
0 & c x
\end{array}\right] \text { and }\left[\begin{array}{cc}
c & 0 \\
0 & c
\end{array}\right]\left[\begin{array}{cc}
x & 0 \\
0 & x
\end{array}\right]=\left[\begin{array}{cc}
c x & 0 \\
0 & c x
\end{array}\right]
$$

for every $x \in N$.

Note that $M$ is not a right Boolean nearring. Also, $M$ is not commutative because

$$
\left[\begin{array}{ll}
b & 0 \\
0 & b
\end{array}\right]\left[\begin{array}{ll}
c & 0 \\
0 & c
\end{array}\right]=\left[\begin{array}{ll}
a & 0 \\
0 & a
\end{array}\right] \neq\left[\begin{array}{ll}
0 & 0 \\
0 & 0
\end{array}\right]=\left[\begin{array}{ll}
c & 0 \\
0 & c
\end{array}\right]\left[\begin{array}{ll}
b & 0 \\
0 & b
\end{array}\right]
$$

Example 1.5. Let $M=\left\{\left[\begin{array}{ll}x & 0 \\ 0 & x\end{array}\right] \mid x \in N\right\}$, where $N=\{0, a, b, c\}$ and + and $\cdot$ are defined on $N$ as follows:

\begin{tabular}{|c|c|c|c|c|}
\hline+ & 0 & $a$ & $b$ & $c$ \\
\hline 0 & 0 & $a$ & $b$ & $c$ \\
\hline$a$ & $a$ & 0 & $c$ & $b$ \\
\hline$b$ & $b$ & $c$ & 0 & $a$ \\
\hline$c$ & $c$ & $b$ & $a$ & 0 \\
\hline
\end{tabular}

\begin{tabular}{|c|c|c|c|c|}
\hline$\cdot$ & 0 & $a$ & $b$ & $c$ \\
\hline 0 & 0 & 0 & 0 & 0 \\
\hline$a$ & 0 & 0 & 0 & 0 \\
\hline$b$ & 0 & $a$ & $b$ & $b$ \\
\hline$c$ & 0 & $a$ & $b$ & $b$ \\
\hline
\end{tabular}

Then $M$ is a right Boolean nearring with $n=\left[\begin{array}{ll}b & 0 \\ 0 & b\end{array}\right]$ because

$$
\left[\begin{array}{ll}
x & 0 \\
0 & x
\end{array}\right]\left[\begin{array}{cc}
x & 0 \\
0 & x
\end{array}\right]=\left[\begin{array}{cc}
x^{2} & 0 \\
0 & x^{2}
\end{array}\right]=\left[\begin{array}{cc}
x b & 0 \\
0 & x b
\end{array}\right] \text { and }\left[\begin{array}{cc}
x & 0 \\
0 & x
\end{array}\right]\left[\begin{array}{cc}
b & 0 \\
0 & b
\end{array}\right]=\left[\begin{array}{cc}
x b & 0 \\
0 & x b
\end{array}\right]
$$

for every $x \in N$. Note that $M$ is not a left Boolean nearring. Also, $M$ is not commutative because

$$
\left[\begin{array}{ll}
a & 0 \\
0 & a
\end{array}\right]\left[\begin{array}{ll}
b & 0 \\
0 & b
\end{array}\right]=\left[\begin{array}{ll}
0 & 0 \\
0 & 0
\end{array}\right] \neq\left[\begin{array}{cc}
a & 0 \\
0 & a
\end{array}\right]=\left[\begin{array}{ll}
b & 0 \\
0 & b
\end{array}\right]\left[\begin{array}{ll}
a & 0 \\
0 & a
\end{array}\right] .
$$

Proposition 1.6. Let $R$ be a left(right) Boolean ring. If $n$ is not a zero divisor then $R$ is commutative.

Proof. Let $\mathrm{R}$ be a left Boolean ring. We have $(x+y)^{2}=n(x+y)$. Then

$$
\begin{aligned}
(x+y)(x+y) & =n x+n y \Rightarrow x(x+y)+y(x+y)=n x+n y \Rightarrow x^{2}+x y+y x+y^{2}= \\
& =n x+n y \Rightarrow n x+x y+y x+n y=n x+n y \Rightarrow x y+y x=0 \Rightarrow x y=-y x .
\end{aligned}
$$

Also,

$$
(x+x)^{2}=n(x+x) \Rightarrow x^{2}+x^{2}+x^{2}+x^{2}=n x+n x \Rightarrow n(x+x)=0 \Rightarrow x=-x .
$$

By (1) and (2) we get, $x y=y x$. The proof is similar for right Boolean ring.

Proposition 1.7. If $N$ is a Boolean nearring with left (resp. right) identity then $N$ is a left (resp. right) Boolean nearring. 
Proof. Let $e$ be a left identity of $N$. Then $x^{2}=x=e x$. Hence $N$ is a left Boolean nearring. Now, let $e$ be a right identity of $N$. Then $x^{2}=x=x e$. Hence $N$ is a right Boolean nearring. $\square$

Example 1.8. Let $N=\{0, a, b, c\}$ with operations + and $\cdot$ defined as follows:

\begin{tabular}{|c|c|c|c|c|}
\hline+ & 0 & $a$ & $b$ & $c$ \\
\hline 0 & 0 & $a$ & $b$ & $c$ \\
\hline$a$ & $a$ & 0 & $c$ & $b$ \\
\hline$b$ & $b$ & $c$ & 0 & $a$ \\
\hline$c$ & $c$ & $b$ & $a$ & 0 \\
\hline
\end{tabular}

\begin{tabular}{|c|c|c|c|c|}
\hline$\cdot$ & 0 & $a$ & $b$ & $c$ \\
\hline 0 & 0 & 0 & 0 & 0 \\
\hline$a$ & 0 & $a$ & $a$ & $a$ \\
\hline$b$ & 0 & $b$ & $b$ & $b$ \\
\hline$c$ & 0 & $c$ & $c$ & $c$ \\
\hline
\end{tabular}

Then $N$ is a non-commutative Boolean nearring. Note that $a, b, c$ are right identities of $N$. By Proposition 1.7, $N$ is a right Boolean nearring for $n \in\{a, b, c\}$. Note that $a, b, c$ are not zero divisors of $N$. This example also shows that Proposition 1.6 is not true in general for left (resp. right) Boolean nearrings.

Proposition 1.9. Let $R$ be a left(resp. right) Boolean ring with $|R| \geqslant 3$. If $0 \neq n$ is not a zero divisor then $R$ has a proper zero divisor.

Proof. Let $x, y \in R$ such that $0 \neq x \neq y \neq 0$. Note that $x+y \neq 0$. If $x y=0$ then $x$ is a proper zero divisor. Let $x y \neq 0$. $(x y)(x+y)=x y x+x y y=y x^{2}+x y^{2}$ (Since $R$ is commutative when $n$ is not a zero-divisor) $=y n x+x n y=x n y+x n y=0$. Thus $x y$ is a proper zero-divisor. The proof is similar for right Boolean ring.

Proposition 1.10. Let $f: R_{1} \rightarrow R_{2}$ be a onto ring homomorphism. If $R_{1}$ is a left(resp. right) Boolean ring then $R_{2}$ is a left(resp. right) Boolean ring.

Proof. Let $R_{1}$ be a left Boolean ring. Let $y \in R_{2}$. Then $y=f(x)$ for some $x \in R_{1}$. Now, $y^{2}=f(x) f(x)=f\left(x^{2}\right)=f(n x)=f(n) f(x)=f(n) y$. Hence $R_{2}$ is a left Boolean ring. Similarly, if $R_{1}$ is a right Boolean ring then $R_{2}$ is a right Boolean ring.

Proposition 1.11. Let $R$ be a field and a left(resp. right) Boolean ring. Then $n=1$.

Proof. Let $n \neq 1$. Now, $x^{2}=n x \Rightarrow x^{2}-n x=0 \Rightarrow x(x-n)=0 \Rightarrow x-n=0 \Rightarrow x=n$. This shows that $n$ varies with each $x$, a contradiction. Hence $n=1$. The proof is similar for right Boolean ring.

Theorem 1.12. Let $N$ be a left(right) Boolean nearring and $P$ be a c-prime ideal of $N$. If $n$ and $m$ are not zero divisors then $P$ is maximal.

Proof. Let $N$ be a left Boolean nearring. Then we have, $x^{2}=n x$. This implies $x^{2}-n x=0 \in$ $P \Rightarrow x(x-n x) \in P \Rightarrow x \in P$ or $x-n x \in P \Rightarrow x \in P$ or $x \in n x+P \Rightarrow N / P=\{P, n x+P\} \Rightarrow N / P$ is a field. Hence $P$ is maximal. The proof is similar for right Boolean nearring exists.

Definition 1.13 (Pilz [24]). A nearring $N$ is said to have insertion of factors property(IFP) if for all $a, b \in N, a b=0$ implies anb $=0$ for all $n \in N$.

Corollary 1.14. Let $N$ be a left(right) Boolean nearring and $P$ be an equiprime ideal that has IFP. If $n$ and $m$ are not zero divisors and $P$ be an equiprime then $P$ is maximal.

Proof. The proof follows from Theorem 2.21 of Kedukodi, Kuncham and Bhavanari [11] and Theorem 1.12.

Definition 1.15. Let I be an ideal of N. I is called left(resp. right) Boolean type if there exists $n \in N$ such that $x^{2}-n x \in I$ (resp. $x^{2}-x n \in I$ ) for all $x \in N$. 
Proposition 1.16. Let I be an ideal of $N$. Then $N / I$ is a left(resp. right) Boolean ring if and only if I is left(resp. right) Boolean type.

Proof. $I$ is left Boolean type $\Leftrightarrow x^{2}-n x \in I \Leftrightarrow x^{2}+I=n x+I \Leftrightarrow(x+I)^{2}=(n+I)(x+I)$. Hence $N / I$ is a left Boolean ring. Similarly, we can prove that $N / I$ is a right Boolean ring if and only if $I$ is right Boolean type.

Note 1.17. If $I=\{0\}$ is left (resp. right) Boolean type then $N$ is a left (resp. right) Boolean nearring.

Example 1.18. Let $R=\{0, a, b, c\}$ with operations + and $\cdot$ defined as follows:

\begin{tabular}{|c|c|c|c|c|}
\hline+ & 0 & $a$ & $b$ & $c$ \\
\hline 0 & 0 & $a$ & $b$ & $c$ \\
\hline$a$ & $a$ & 0 & $c$ & $b$ \\
\hline$b$ & $b$ & $c$ & 0 & $a$ \\
\hline$c$ & $c$ & $b$ & $a$ & 0 \\
\hline
\end{tabular}

\begin{tabular}{|c|c|c|c|c|}
\hline$\cdot$ & 0 & $a$ & $b$ & $c$ \\
\hline 0 & 0 & 0 & 0 & 0 \\
\hline$a$ & 0 & $a$ & 0 & $a$ \\
\hline$b$ & 0 & 0 & $b$ & $b$ \\
\hline$c$ & 0 & $a$ & $b$ & $c$ \\
\hline
\end{tabular}

Then $R$ is a left (resp. right) Boolean ring with $n=c$ and left identity $c$.

Theorem 1.19. Let $R$ be a left(resp. right) Boolean nearring. If $n$ is a distributive element and has a left identity e, then $R$ is a zero symmetric nearring. Further, if there exists a left ideal $I$ such that $e \in I$ and (i) $n x \in I \Rightarrow x \in I$ (resp. $x n \in I \Rightarrow x \in I$ ); (ii) $[X, Y] \cap I=\{0\}$ then $R$ is a commutative ring.

Proof. As $e$ is left distributive, $(e+e)^{2}=n(e+e)=n e+n e$. Now, $(e+e)^{2}=(e+e)(e+e)=$ $=e(e+e)+e(e+e)=e^{2}+e^{2}+e^{2}+e^{2}=n e+n e+n e+n e \Rightarrow n e+n e=0 \Rightarrow n(e+e)=0 \Rightarrow e+e=0$. If $x$ is in $R$, then $x+x=(e+e) x=0 . x=0 \Rightarrow x+x=0$. Let $w$ be arbitrary element in $R$. Then $(e+w)^{2}=n(e+w) \Rightarrow(e+w)(e+w)=n e+n w \Rightarrow e(e+w)+w(e+w)=n e+n w \Rightarrow$ $e^{2}+w+w(e+w)=n e+n w \Rightarrow w(e+w)=-w+n w=w+w^{2}=(e+w) w$.

$$
w(e+w)-(e+w) w=0 .
$$

Now, $w(e+w) 0=(e+w) w 0 \Rightarrow w(e 0+w 0)=e w 0+w w 0 \Rightarrow w w 0=e w 0+w w 0 \Rightarrow w 0=0$. Thus $R$ is a zero symmetric nearring. Replacing $w=a b$ and $w=b a$ in equation (3), we get $(e+a b) a b=a b(e+a b) \Rightarrow a b+(a b)^{2}=a b(e+a b)$. Now, we have

$$
a b=a b(e+a b)-n a b .
$$

Similarly, $b a=b a(e+b a)-n b a$.

Hence $a b-b a=a b(e+a b)-n a b-[b a(e+b a)-n b a] \in I$. We have $a b-b a \in[X, Y]=\{x y-y x \mid x \in$ $X, y \in Y\}$ and $a b-b a \in I \Rightarrow a b-b a=0 \Rightarrow a b=b a$. Proof is similar for right Boolean ring.

Corollary 1.20. Let $R$ be a left(resp. right) Boolean nearring. Let $n$ be a distributive non zero divisor of $R$ and $I$ be a left ideal such that $[X, Y] \cap I=\{0\}$. Then $(R, \leqslant)$ is a partially ordered set with $\leqslant$ defined by $x \leqslant y$ if $x y=n x$ (resp. $x y=x n)$. Further, if $n x \in I \Rightarrow x \in I$ (resp. $x n \in I \Rightarrow x \in I)$ and $R$ has a left identity e such that $e \in I$ then $(R, \leqslant)$ is a lattice with meet and join operations given respectively by $x \wedge y=x y$ and $x \vee y=x+y+x y$.

Proof. It is straightforward to verify that $(R, \leqslant)$ is a partially ordered set. The rest of the proof follows from Theorem 1.19.

Definition 1.21. Let $N$ be a left(resp. right) Boolean nearring and I be an ideal of $N . N$ is said to satisfy weak commutative property with respect to ideal I if for all $a, b, c \in N, a b c-a c b \in I$. 
Lemma 1.22. Let $N$ be a left(resp. right) Boolean nearring. If there exists an ideal I of $N$ such that (i) $n x \in I \Rightarrow x \in I$ (resp. $x n \in I \Rightarrow x \in I$ ), and (ii) $x I x \subseteq I$ for all $x \in N$, then $a b-a b a \in I$ for all $a, b \in I$.

Proof. We have $(a b-a b a)^{2}=n(a b-a b a)$. Now, $(a b-a b a)^{2}=(a b-a b a)(a b-a b a)=$ $=a b(a b-a b a)-a b a(a b-a b a)=a b\left(a b-i_{1}\right)-i_{2}\left[\right.$ where $i_{1}=a b a$ and $\left.i_{2}=a b a(a b-a b a)\right]=$ $=i_{3}+a b a b-i_{4} \in I$. Hence we get $n(a b-a b a) \in I$. This implies $a b-a b a \in I$. The proof is similar for right Boolean ring.

Theorem 1.23. Let $N$ be left(resp. right) Boolean nearring. If there exists an ideal I in $N$ such that (i) $n x \in I \Rightarrow x \in I$ (resp. $x n \in I \Rightarrow x \in I$ ) and (ii) $x I x \subseteq I$ for all $x \in N$, then $N$ satisfies weak commutative property with respect to $I$.

Proof. We have $a b c-a c b=a b c-a\left(c b c+i_{1}\right)$ [because $c b-c b c \in I$ implies $c b=c b c+i_{1}$ ] $=a b c-i_{2}-a c b c=a b c-a c b c-i_{2}=(a-a c) b c-i_{2}=\left((a-a c) b(a-a c)+i_{3}\right) c-i_{2} \in I$.

Definition 1.24 (Plasser [26]). A nearring $N$ has strong IFP if and only if for all ideals $I$ of $N$ and for all $a, b, n \in N, a b \in I$ implies $a n b \in I$.

Corollary 1.25. Let $N$ be a left (resp. right) Boolean nearring with an ideal $I$ such that (i) $n x \in I \Rightarrow x \in I$ (resp. $x n \in I \Rightarrow x \in I$ ) and (ii) $x I x \subseteq I$ for all $x \in N$. Then $N$ has strong IFP.

Proof. Let $a b \in I$. By Theorem 2.4, $a x b=a b x+i \in I$. This implies $N$ has strong IFP.

Theorem 1.26. Let $N$ be a left(resp. right) Boolean nearring and $I$ be an ideal of $N$ such that (i) $n x \in I \Rightarrow x \in I$ (resp. $x n \in I \Rightarrow x \in I$ ); (ii) $x I x \subseteq I$ for all $x \in N$. If $L$ is any left ideal of $N$ containing $I$ then $L$ is an ideal of $N$.

Proof. Let $L$ be a left ideal of $N$. To show that $L$ is an ideal it suffices to show that $L N \subseteq L$. Let $l \in L, n \in N$. Then we have $l=l_{0}+l_{c}$ and $n=n_{0}+n_{c}$ as Pierce decompositions, where $l_{0}, n_{0} \in N_{0} ; l_{c}, n_{c} \in N_{c}-(4)$. As $L$ is a left ideal, we have $N_{0} L \subseteq L$, for $m_{0} l^{\prime}=$ $=m_{0}\left(0+l^{\prime}\right)-m_{0} 0 \in L$ for all $m_{0} \in N_{0}, l \in L-(5)$. Now, $l n=l^{2}=\left(l_{0}+l_{c}\right) l=l_{0} l+l_{c} l=l_{0} l+l_{c}$. By $(5), l, l_{0} l \in L$ and it follows that $l_{c} \in L$ and hence $l_{0} \in L$. We have, $l n=\left(l_{0}+l_{c}\right) n=$ $=l_{0} n+l_{c} n=l_{0} n+l_{c}=l_{0}\left(n_{0}+n_{c}\right)+l_{c}=l_{0}\left(n_{0}+n_{c}\right) l_{0}+i+l_{c}=l_{0}\left(n_{0} l_{0}+n_{c}\right)+i+l_{c}$. We have $l_{0} n_{c}=l_{0} n_{c} 0=l_{0} 0 n_{c}+i=0+i \in I$. As $n_{0}, l_{0} \in L$, we have $l_{0}\left(n_{0} l_{0}+n_{c}\right)=l_{0} n_{c}+i_{2} \in I \subseteq L$. Hence $l n=l_{0}\left(n_{0} l_{0}+n_{c}\right)+l_{c} \in L$. Thus $L$ is an ideal of $\mathrm{N}$.

\section{Derivations}

Definition 2.1 (Bell [1]). A derivation on $N$ is defined to be an additive endomorphism satisfying the product rule $D(x y)=x D(y)+D(x) y$ for all $x, y \in N$.

Proposition 2.2. Let $N$ be a 3-prime, 2 torsion left(resp. right) Boolean nearring. If $D$ is a commuting derivation on $N$, then either $D(n)=0$ or $D(n)=n$.

Proof. Let $N$ be 3-prime left Boolean nearring. Then $x^{2}=n x$. We have $D\left(x^{2}\right)=D(n x) \Rightarrow$ $x D(x)+D(x) x=n D(x)+D(n) x$. As $D$ is commuting and $x+x=0$, we get $n D(x)+D(n) x=0$. Put $x=n$, we have $n D(n)+D(n) n=0 \Rightarrow(D(n))^{2}+D(n) n=0 \Rightarrow D(n)(D(n)+n)=0$. Hence $D(n)=0$ or $D(n)=n$. The proof is similar for right Boolean nearring.

Proposition 2.3. Let $N$ be 2 torsion left(resp. right) Boolean nearring. If $D$ is a commuting derivation on $N$ with $D(n)=n$, then $x^{2}+y^{2}=(D(x))^{2}+(D(y))^{2}$ for all $x, y \in N$. 
Proof. Let $N$ be a left Boolean nearring. Then we have $(x+y)^{2}=n(x+y)$. This gives $D(x+y)(x+y)=D(n(x+y)) \Rightarrow(x+y) D(x+y)+D(x+y)(x+y)=D(n(x+y)) \Rightarrow$ $(x+y) D(x+y)+D(x+y)(x+y)=n D(x+y)+D(n)(x+y) \Rightarrow((x+y)+(x+y)-n) D(x+y)=$ $=n(x+y) \Rightarrow n D(x+y)=x^{2}+y^{2} \Rightarrow x^{2}+y^{2}=n D(x)+n D(y)=(D(x))^{2}+(D(y))^{2}$. The proof is similar for right Boolean nearring.

Theorem 2.4. Let $R$ be a left(resp. right) Boolean ring and $D$ be the derivation on $R$ with $D(n x)=0$ (resp. $D(x n)=0)$ for all $x \in R$. Then $D(x y)=-D(y x)$.

Proof. Let $R$ be left Boolean ring. Then $x^{2}=n x$. We have $D\left(x^{2}\right)=D(n x)=0$. $\Rightarrow$ $x D(x)+D(x) x=0 \Rightarrow D(x) x=-x D(x)$. Now we have $(x+y)^{2}=n(x+y)$. Then $D(x+y)^{2}=$ $=D(n(x+y))=0 \Rightarrow D((x+y)(x+y))=0 \Rightarrow(x+y) D(x+y)+D(x+y)(x+y)=0 \Rightarrow$ $x D(x)+x D(y)+y D(x)+y D(y)+D(x) x+D(y) x+D(x) y+D(y) y=0 \Rightarrow x D(y)+y D(x)+$ $+D(y) x+D(x) y=0 \Rightarrow D(x y)=-D(y x)$. The proof is similar for right Boolean nearring.

Corollary 2.5. 1. If $x+x=0$, then $D(x y)=D(y x)$.

2. If $R$ is a zero square ring, then $D(x y)=-D(y x)$.

Proposition 2.6. Let $N$ be left Boolean nearring without zero divisors. Let $D$ be the derivation on $N$. Then $D(n)=n$ or $D(n)=0$.

Proof. We have $x^{2}=n x$. Then $(D(n))^{2}=n D(n) \Rightarrow D(n) D(n)=n D(n)$. Then $(D(n)-$ $-n) D(n)=0 \Rightarrow D(n)=n$ or $D(n)=0$.

Proposition 2.7. Let $N$ be right Boolean nearring without zero divisors. Let $D$ be the commuting derivation on $N$. Then $D(n)=n$ or $D(n)=0$.

Proof. We have $x^{2}=x n$. Then $(D(n))^{2}=D(n) n \Rightarrow D(n) D(n)=D(n) n$. Then $(D(n)-$ $-n) D(n)=0 \Rightarrow D(n)=n$ or $D(n)=0$.

Proposition 2.8. Let $N$ be a left Boolean nearring. If there exists a nilpotent element in $N$, then $n 0=0$.

Proof. Let $x$ be a nilpotent element in $N$. Then we have $x^{k}=0$ for some $k \in \mathbb{N}$. As $N$ is left Boolean nearring, we have $x^{2}=n x$ for $n \in N$. Now, $\left(x^{k}\right)^{2}=n x^{k}=n 0 \Rightarrow n 0=\left(x^{k}\right)^{2}=0$.

Proposition 2.9. Let $D$ be the derivation on left Boolean nearring $N$ such that $D_{k}(x)=k x$. If $n$ is not a zero divisor then $D_{k}(x)=0$.

Proof. We have $D_{k}\left(x^{2}\right)=k x^{2}=k n x \Rightarrow D_{k}(n x)=k n x \Rightarrow n D_{k}(x)+D_{k}(n) x=k n x \Rightarrow$ $n k x+k n x=k n x$. Hence $n k x=0$. As $n$ is not a zero divisor, we get $k x=D_{k}(x)=0$.

Proposition 2.10. Let $N$ be a nearring. If $D_{k}(x)=k x$ is a nonzero derivation on $N$ then $N k N=\{0\}$.

Proof. As $D$ is a derivation on $N$, we have $D_{k}(x y)=x D_{k}(y)+D_{k}(x) y$. Hence $k x y=$ $=x k y+k x y \Rightarrow x k y=0 \Rightarrow N k N=\{0\}$.

Proposition 2.11. Let $N$ be a left Boolean nearring such that $x N y=0$ for all $x$ and $y$. If $n$ is a distributive element then $D(x)=x^{2}$ is a derivation on $N$.

Proof. We have $D(x)=x^{2}=n x$. Now, $D(x+y)=n(x+y)=n x+n y=D(x)+D(y)$. We have $D(x y)=n x y$. Now $x D(y)+D(x) y=x y^{2}+x^{2} y=x n y+n x y=0+n x y=n x y \Rightarrow D(x y)=$ $=x D(y)+D(x) y$. Hence $D$ is a derivation on $N$.

Proposition 2.12. Let $N$ be a right Boolean nearring. If $x N y=0$ for all $x$ and $y$ then $D(x)=x^{2}$ is a derivation on $N$. 
Proof. We have $D(x)=x^{2}=x n$. Now, $D(x+y)=(x+y) n=x n+y n=D(x)+D(y)$. We have $D(x y)=x y n$. Now $x D(y)+D(x) y=x y^{2}+x^{2} y=x y n+x n y=x y n+0=x y n \Rightarrow D(x y)=$ $=x D(y)+D(x) y$. Hence $D$ is a derivation on $N$.

Theorem 2.13. Let $R$ be a ring, $D$ is a derivation on $R$ and $k \in R$. Then $D_{k}(x)=D(x)+k x$ is a derivation on $R$ if and only if $R k R=\{0\}$.

Proof. Let $R k R=\{0\}$. Now, $D_{k}(x+y)=D(x+y)+k(x+y)=D(x)+D(y)+k x+k y=D(x)+$ $+k x+D(y)+k y==D_{k}(x)+D_{k}(y)$. We have $D_{k}(x y)=D(x y)+k x y=x D(y)+D(x) y+k x y$. Now, $x D_{k}(y)+D_{k}(x) y=x(D(y)+k y)+(D(x)+k x) y=x D(y)+x k y+D(x) y+k x y=$ $=x D(y)+D(x) y+k x y \Rightarrow D_{k}(x y)=x D_{k}(y)+D_{k}(x) y$. Hence $D_{k}(x)=D(x)+k x$ is a derivation on $R$.

Conversely, let $D_{k}(x)=D(x)+k x$ be a derivation on $R$. We have $D_{k}(x y)=D(x y)+$ $+k x y=x D(y)+D(x) y+k x y$. Now, $x D_{k}(y)+D_{k}(x) y=x(D(y)+k y)+(D(x)+k x) y=$ $=x D(y)+x k y+D(x) y+k x y$. As $D_{k}(x y)=x D_{k}(y)+D_{k}(x) y$, we have $x D(y)+D(x) y+k x y=$ $=x D(y)+x k y+D(x) y+k x y \Rightarrow x k y=0$. Hence $R k R=\{0\}$.

Theorem 2.14. Let $R$ be a 3-prime left(resp. right) Boolean ring. If $D$ is the derivation on $R$ with $D(n x)=0$ (resp. $D(x n)=0$ ) for all $x \in R$ then $R$ is a commutative ring.

Proof. The proof follows from Corollary 3.4 (ii) of Kamal and Al-Shaalan [10] and Theorem 2.4.

Corollary 2.15. Let $R$ be a 3-prime left(resp. right) Boolean ring with $x+x=0$ for all $x \in R$. If $D$ is the derivation on $R$ such that $D(n x)=0$ (resp. $D(x n)=0$ ) then $R$ is a commutative ring.

Proof. The proof follows from Corollary 2.2 of Kamal and Al-Shaalan [10] and (1) of Corollary 2.5 .

The authors thank the anonymous reviewers for their comments and suggestions. All authors acknowledge Manipal Institute of Technology, Manipal Academy of Higher Education for their encouragement. The first author acknowledges Manipal Academy of Higher Education for Dr. T. M.A. Pai PhD Scholarship.

\section{References}

[1] H.E.Bell, G.Mason, On Derivations in Near-rings, Proceeding of Near-rings and Near-Fields, Tubigen, 1985, North-Holland Mathematical Studies, 31-35, 1987.

[2] S.Bhavanari, S.P.Kuncham, Linearly Independent Elements in N-Groups with Finite Goldie Dimension, Bull. Korean Math. Soc., 42(2005), 433-441.

[3] S.Bhavanari, S.P.Kuncham, Nearring, Fuzzy Ideals and Graph Theory, CRC press (UK, USA), 2013.

[4] S.Bhavanari, Contributions to Near-rings, VDM Verlag Dr Mullar, Germany, 2010.

[5] S.Bhavanari, S.P.Kuncham, B.S.Kedukodi, Graph of a Nearring with respect to an Ideal, Comm. Algebra, 38(2010), 1957-1962.

[6] S.Bhavanari, L.Godloza, M.Babu Prasad, S.P.Kuncham, Ideals and Direct Products of Zero-square Nearrings, Int. J. Algebra (Croatia), 4(2010), 13-16. 
[7] R.James Clay, A.L.Donald, Boolean Near-ring, Canad. Math. Bull., 12(1969), 265-273.

[8] B.Jagadeesha, S.P.Kuncham, B.S.Kedukodi, Implications on a Lattice, Fuzzy Inf. Eng., 8(2016), no. 4, 411-425.

[9] B.Jagadeesha, B.S.Kedukodi, S.P.Kuncham, Interval valued L-fuzzy ideals based on t-norms and t-conorms, J Intell. Fuzzy Syst., 28(6)(2016), 2631-2641

[10] A.A.M Kamal, K.H.Al-Shaalan, Commutativity of Near-rings with Derivations by using Algebraic Substructures, Indian J. Pure Appl. Math., 43(2012), no. 3, 211-225.

[11] B.S.Kedukodi, S.P.Kuncham, S.Bhavanari, Equiprime, 3-prime and c-prime Fuzzy Ideals of Nearrings, Soft. Comput., 13(2009), 933-944.

[12] B.S.Kedukodi, S.P.Kuncham, S.Bhavanari, C-prime Fuzzy Ideals of Nearrings, Soochow J. Math., 33(2007), 891-901.

[13] B.S.Kedukodi, B.Jagadeesha, S.P.Kuncham, S.Juglal, Different Prime Graphs of a Nearring with respect to an Ideal, 185-203, Editors: S.P.Kuncham, B.S.Kedukodi, H.Panackal, S. Bhavanari, Nearrings, Nearfields and Related Topics, World Scientific Publisher, Singapore, 2017.

[14] B.S.Kedukodi, S.P.Kuncham and S.Bhavanari, Reference points and roughness, Inform. Sci., 180(2010), no. 17, 3348-3361.

[15] B.S.Kedukodi, S.P.Kuncham and S.Bhavanari, C-Prime fuzzy ideals of Rn[x], Int. J Contemp. Math. Sci., 8(2013), no. 3, 133-137.

[16] B.S. Kedukodi, S.P.Kuncham and B.Jagadeesha, Interval valued L fuzzy prime ideals, triangular norms and partially ordered groups, Soft Comput., (2017), https://doi.org/10.1007/s00500-017-2798-x.

[17] K.Koppula, B.S.Kedukodi, S.P.Kuncham, Markov chains and rough sets, Soft. Comput., (2018), https://doi.org/10.1007/s00500-018-3298-3.

[18] S.P.Kuncham, Contributions to Near-ring Theory II, Doctoral Dissertation Acharya Nagarjuna University, 2000.

[19] S.P.Kuncham, B.Jagadeesha, B.S.Kedukodi, Interval valued L-fuzzy cosets of nearrings and isomorphism theorems, Afrika Mat., 27(2016), no. 3, 393-408.

[20] S.P.Kuncham, B.S.Kedukodi, H.Panackal and S.Bhavanari, Nearrings, Nearfields and Related Topics, World Scientific, Singapore, 2017 (ISBN: 978-981-3207-35-6).

[21] B.Lafuerza-Guillén, H.Panackal, Probabilistic Normed Spaces, Imperial College Press, World Scientific, UK, London, 2014.

[22] A.Molkhasi, Strongly Algebraically Closed Lattices in l-groups and Semilattices, Journal of Siberian Federal University. Mathematics \& Physics, 11(2018), no. 2, 258-263.

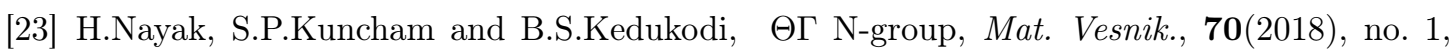
64-78. 
[24] G.Pilz, Near-rings and Near-fields (Handbook of Algebra), Edited by M.Hazewinkel, Elsevier Science B.V., 1996.

[25] H.Panackal, B.Lafuerza-Guillén, K.T.Ravindran, Compactness and $\mathcal{D}$ - boundedness in Menger's 2-Probabilistic Normed Spaces, FILOMAT, 30(2016), no. 5, 1263-1272.

[26] K.Plasser, Subdirekte darstellung von ringen und fastringen mit boolschen eigenzchaften, Diplomarbeit, Univ. Linz, Austria, 1974.

[27] Y.V.Reddy, Recent developments in Boolean Nearrings, Editors: S. P. Kuncham, B.S.Kedukodi, H.Panackal, S.Bhavanari, Nearrings, Nearfields and Related Topics, World Scientific Publisher (Singapore), 2017.

\section{Расширения булевых колец и почти колец}

Хамса Наяк

Сям П. Кунчам

Бабушри С. Кедукоди

Математический факультет

Манипальский технологический институт

Манипальская Академия высшего образования

Манипал, Карнатака, 576104

Индия

В этой статъе мы вводим понятия левых (правых) булевых колец, и почти колец. Мы приводим примеры, чтобы показать, что левые (правые) булевы кольца вообще не коммутативны. Мы получаем взаимосвязи между этими алгебраическими структурами и получаем условия, при которых структуры являются коммутативными. Наконеи, мы изучаем концепиию дифференцирования на левых (правых) булевых кольцах и почти кольцах и получаем результаты коммутативности.

Ключевые слова: кольцо, почти кольцо, булево кольцо, булево почти кольцо. 